TRANSACTIONS OF THE

AMERICAN MATHEMATICAL SOCIETY

Volume 295, Number 2, June 1986

\title{
CONNECTED LOCALLY CONNECTED TOPOSES ARE PATH-CONNECTED
}

\author{
I. MOERDIJK ${ }^{1}$ AND G. C. WRAITH
}

\begin{abstract}
A conjecture of A. Joyal is proved, which states that, in contrast to topological spaces, toposes which are connected and locally connected are also path-connected. The reason for this phenomenon is the triviality of cardinality considerations in the topos-theoretic setting; any inhabited object pulls back to an enumerable object under some open surjective geometric morphism. This result points towards a homotopy theory for toposes.
\end{abstract}

Introduction. The proposition stated by the title was conjectured by A. Joyal in 1983 during a seminar at Columbia University. Every topologist knows that a connected locally connected topological space is not necessarily path-connected. The set of natural numbers with the cofinite topology is an example, and so is "the long segment". However, it is true that all connected locally connected complete metric spaces are path-connected (Menger (1929), Moore (1932)).

Toposes are generalizations of (sober) topological spaces if we identify a topological space $X$ with the topos of sheaves on $X$. The notions of connectedness and local connectedness were defined in SGA 4 (Grothendieck and Verdier (1972)) for toposes in a way that extends the usual versions of these concepts for topological spaces. How then can Joyal's conjecture be true? The explanation lies in the correct interpretation of what path-connectedness means for a topos $\mathscr{E}$. It does not mean that "for every pair of points $x_{0}, x_{1}$ of $\mathscr{E}$ there is a path $I \stackrel{f}{\rightarrow} \mathscr{E}$ with $f(0)=x_{0}$, $f(1)=x_{1}$ ". This is an inappropriate definition, inasmuch as toposes do not necessarily have points. Instead, one has to construct the "space of paths in $\mathscr{E}$ " as again being a topos. More precisely, a topos $\mathscr{F}$ is exponentiable if the 2-functor $\mathscr{F} \times(-)$ has a right 2 -adjoint $(-)^{\mathscr{F}}$, and $\mathscr{E}^{\mathscr{F}}$ is interpretable as the topos of maps from $\mathscr{F}$ to $\mathscr{E}$. Points of $\mathscr{E}^{\mathscr{F}}$ correspond to maps from $\mathscr{F}$ to $\mathscr{E}$. The (topos of sheaves on the) unit interval $I$ is an exponentiable topos, so for any topos $\mathscr{E}$ we may form the topos $\mathscr{E}^{I}$ of paths in $\mathscr{E}$. The inclusion of the endpoints $\{0,1\} \rightarrow I$ induces a map of toposes $\mathscr{E}^{I} \rightarrow \mathscr{E} \times \mathscr{E}$, and it is natural to say that $\mathscr{E}$ is path-connected if this map is a surjection. We will prove the following slightly stronger result (over an arbitrary base topos).

Received by the editors October 3, 1984.

1980 Mathematics Subject Classification (1985 Revision). Primary 18B25: Secondary 54D05.

${ }^{1}$ Supported by the Netherlands Organisation for the Advancement of Pure Research (ZWO). 
THEOREM. For any connected locally connected topos $\mathscr{E}$, the canonical map $\mathscr{E}^{I} \rightarrow$ $\mathscr{E} \times \mathscr{E}$ is an open surjection; so, in particular, $\mathscr{E}$ is path-connected.

The explanation of what "goes wrong" for spaces like the long segment $L$ is straightforward. The topos $L^{I}$ has no points corresponding to nontrivial paths reaching the endpoint (see e.g. Steen and Seebach (1978), Engelking (1977)), but $L^{\prime} \rightarrow L \times L$ is nevertheless a surjective map of toposes.

In attempting to prove a result of this kind, two approaches are available. One is to manipulate directly with a site for $\mathscr{E}^{I}$ (or a suitable site "covering" this topos). In this rather algebraic approach, one generally "stays at one place" (one base topos). The other approach is more geometrical: the strategy is to use adequate extensions of the base topos available from general topos theory, which enable one to follow classical arguments about points of separable metric spaces rather closely. Although both approaches are equivalent, we will follow the second one, because it shows more clearly the interplay between general topos theory and arguments (somewhat similar to those) from topology. (But we will also give a brief description of the maps of sites involved in the "algebraic approach"; see 2.6 below.)

Apart from the element of surprise, and as an illustration of the slogan that generalized spaces are better behaved than topological spaces, what can this result be used for? One answer is: homotopy theory for toposes. Homotopy groups of topological spaces are really topological groups (which usually, but not always, turn out to be discrete), so it is hardly revolutionary to insist that homotopy groups (or groupoids, or other gadgets) of toposes are themselves toposes. The point is made in SGA 4 that the right notion of quotient by an equivalence relation for toposes is to take the topos of descent data. If $\mathscr{X}$. denotes a simplicial topos, $\Pi_{0}(\mathscr{X}$.) will denote the topos of descent data; that is, its objects are pairs $(A, \sigma)$, where $A$ is an object of $\mathscr{X}_{0}$ and $\sigma$ is an isomorphism $d_{0}^{*} A \rightarrow d_{1}^{*} A$ in $\mathscr{X}_{1}$ satisfying the usual coherence conditions. We have a surjective map of toposes $\mathscr{X}_{0} \rightarrow \Pi_{0}\left(\mathscr{X}_{0}\right)$. Let $\Delta$. denote the cosimplicial topos given by the standard simplices. For any topos $\mathscr{E}$, we have the simplicial topos $\mathscr{E}^{\Delta .}$, and we define $\pi_{0}(\mathscr{E})$ to be the topos $\Pi_{0}\left(\mathscr{E}^{\Delta .}\right)$. This is the topos of connected components of $\mathscr{E}$.

Of course, $\Delta_{1}$ is just the unit interval $I$. Let us denote by $P(\mathscr{E})$ the $\mathscr{E} \times \mathscr{E}$-topos $\mathscr{E}^{\prime} \rightarrow \mathscr{E} \times \mathscr{E}$. We denote by $\Gamma(\mathscr{E})$ the $\mathscr{E} \times \mathscr{E}$-topos $\Gamma_{1}(\mathscr{E}) \rightarrow \mathscr{E} \times \mathscr{E}$ given by $\pi_{0}(P(\mathscr{E}))$, obtained by applying $\pi_{0}$ in the context of $\mathscr{E} \times \mathscr{E}$-toposes. We assert that $\Gamma_{1}(\mathscr{E}) \rightarrow$ $\mathscr{E} \times \mathscr{E}$ is a groupoid topos and is the fundamental groupoid topos of $\mathscr{E}$. Pulling back along the diagonal $\mathscr{E} \rightarrow \mathscr{E} \times \mathscr{E}$ gives the fundamental group $\pi_{1}(\mathscr{E})$ as an $\mathscr{E}$-topos (this takes care of the base point). We hope to say more about this in a later paper.

P. Johnstone has pointed out to us an example of a topological space having a trivial fundamental group as a topological space but a nontrivial fundamental group as a generalized space, a topos. The example is a "long loop" (obtained from the long segment by identifying the two endpoints), which admits no nontrivial maps from the circle, but - being connected and locally connected-has a nontrivial, but pointless, generalized "space" of loops. Since the homotopy relation for it is given by an open equivalence relation, its fundamental group as a generalized space will be discrete and isomorphic to $\mathbf{Z}$. 
Acknowledgments. Both authors would like to thank the organizers of the category theory conference at Löwenberg Manor, where the actual writing of this article got started, and P. Johnstone, A. Joyal and A. Kock for valuable conversations.

1. Preliminaries. In this paper, all toposes are Grothendieck over a fixed, but arbitrary, base topos $\mathscr{S}$ (thought of as "the" category of sets).

1.1 Spaces and locales. Our terminology concerning locales, spaces, etc., will be as in Joyal and Tierney (1984). So a locale is a complete Heyting algebra, and a map of locales is a function which preserves finite meets and arbitrary sups; spaces are the duals of locales. For a space $X, \mathcal{O}(X)$ denotes the corresponding locale, the elements of which are called the opens of $X$. A (sober) topological space is a space with enough points.

A presentation of a space $X$ is a poset $\mathbf{P}$ equipped with a stable system of covering families such that $\mathcal{O}(X)$ is isomorphic to the set of downwards closed subsets of $\mathbf{P}$ which are closed for the system of covers; i.e.,

$$
\mathcal{O}(X) \cong\{S \subseteq \mathbf{P} \mid(p \leqslant q \in S \Rightarrow p \in S) \text {, and ( } T \text { covers } p, T \subseteq S \Rightarrow p \in S)\} .
$$

(Equivalently, $\mathbf{P}$ is a site for the topos of sheaves on $X$.)

For general information about spaces and locales, see Isbell (1972), Johnstone (1982), Joyal and Tierney (1982), and Hyland (1981).

1.2 Open maps. A geometric morphism $\mathscr{F} \stackrel{\varphi}{\rightarrow} \mathscr{E}$ is open if $\varphi^{*}$ preserves first-order logic. $\varphi$ is open iff its localic part (its spatial reflection) is, iff the unique $\wedge \vee$-map $\Omega_{\mathscr{E}} \rightarrow \varphi_{*}\left(\Omega_{\mathscr{F}}\right)$ in $\mathscr{E}$ has an internal left adjoint. (A topos $\mathscr{F}$ is called open if the canonical map $\mathscr{F} \rightarrow \mathscr{S}$ is an open geometric morphism.) An important characterization states that $\mathscr{F} \stackrel{\varphi}{\rightarrow} \mathscr{E}$ is open iff there is a site $\mathbf{C}$ for $\mathscr{F}$ in $\mathscr{E}$ such that (in $\mathscr{E}$ it holds that) all covers in $\mathbf{C}$ are inhabited. We can take $\mathbf{C}$ to have a terminal object iff $\mathscr{F} \rightarrow \mathscr{E}$ is also a surjection. In particular, a space $X$ is open (and surjective) iff it has a presentation $\mathbf{P}$ (with a top-element 1 ) the covers of which are all inhabited.

For some proofs and more information, see Johnstone (1980), Joyal and Tierney (1984).

1.3 Connected locally connected maps. A geometric morphism $\mathscr{F} \stackrel{\varphi}{\rightarrow} \mathscr{E}$ is connected if $\varphi^{*}$ is full and faithful. $\varphi$ is called locally connected (or molecular) if $\varphi^{*}$ commutes with $\Pi$-functors. $\mathscr{F} \stackrel{\varphi}{\rightarrow} \mathscr{E}$ is locally connected iff there is a site $\mathbf{C}$ for $\mathscr{F}$ in $\mathscr{E}$, all whose covers are inhabited and connected, and we may take $\mathbf{C}$ to have a terminal iff $\varphi$ is also connected. In particular, a space $X$ is connected and locally connected iff it has a presentation $\mathbf{P}$ with a top-element 1 whose covers are inhabited and connected. For $\mathbf{P}$ we can take the connected open subspaces of $X$, so we may without loss of generality assume that $\mathbf{P}$ is closed under sups of chains. (A chain in $\mathbf{P}$ is a sequence $\left(V_{1}, \ldots, V_{k}\right)$ of elements of $\mathbf{P}$ such that for each $i=1, \ldots, k-1$, there is a $W_{i} \in \mathbf{P}$ with $W_{i} \leqslant V_{i}, W_{i} \leqslant V_{i+1}$; equivalently, since all covers in $\mathbf{P}$ are inhabited, $V_{i} \wedge V_{i+1}$ is a surjective (open) space.) We call such a presentation $\mathbf{P}$ of $X$, with $1 \in \mathbf{P}, \mathbf{P}$ closed under sups of chains, and all covers inhabited and connected, a molecular presentation of $X$. 
For proofs and further information, see Barr and Paré (1980), and the appendix of Moerdijk (1984).

The properties of being an open (surjective) map and of being a (connected) locally connected map are closed under composition. Moreover, as is clear from the characterizations in terms of sites, these properties are preserved by pulling back along an arbitrary geometric morphism.

1.4 Exponentiability. A topos $\mathscr{F}$ is exponentiable if the functor $\mathscr{F} \times(-)$ of Grothendieck toposes over $\mathscr{S}$ has a right adjoint $(-)^{\mathscr{F}}$ (in the appropriate 2-categorical sense). Any compact regular space $X$ is exponentiable as a topos, and if $Y$ is any space, $Y^{X}$ is the topos of sheaves on the space $Y^{X}$, i.e., the exponential in the category of spaces (so there is no harm in not distinguishing the two notationally). The construction of the exponential space $Y^{X}$ in $\mathscr{S}$ is stable; that is, if $\mathscr{S}^{\prime} \stackrel{\varphi}{\rightarrow} \mathscr{S}$ is a geometric morphism, then $\varphi^{\#}\left(Y^{X}\right) \cong \varphi^{\#}(Y)^{\varphi^{\#}(X)}$ as spaces in $\mathscr{S}^{\prime}$.

For exponentials of toposes see Johnstone and Joyal (1984); the case of spaces is dealt with in Hyland (1981).

1.5 The unit interval. By the unit interval $I$ we will always mean the unit interval as defined as a locale, as a "formal space" (see e.g. Fourman and Grayson (1982)). Thus, in any topos $\mathscr{S}, I$ is a compact regular space, and hence exponentiable as a space and as a topos. Moreover, the construction of $I$ as a formal space is stable; i.e. for a geometric morphism $\mathscr{S}^{\prime} \stackrel{\varphi}{\rightarrow} \mathscr{S}, \varphi^{\#}\left(I_{\mathscr{S}}\right)=I_{\mathscr{S}^{\prime}}$ (where the subscript denotes where $I$ is constructed; by stability, this subscript can be suppressed). ( $I$ need not coincide with the corresponding topological space of Dedekind cuts; in fact it does iff this topological space is compact. Since we work over an arbitrary base topos, we have to deal with the formal space rather than the topological space.)

1.6 Some base extensions. We will use the following three types of base extensions.

Lemma A (JoyAL). Let $\mathscr{E}$ be a given topos over $\mathscr{S}$. Then there exists a space $X$ in $\mathscr{S}$ and a geometric morphism $X \rightarrow \mathscr{E}$ which is connected and locally connected. Thus, if $\mathscr{E}$ is itself connected locally connected, so is $X$.

Proof. See Johnstone (1984).

LEMMA B. Let $\left\{S_{i}\right\}_{i \in I}$ be a (small) family of inhabited objects in $\mathscr{S}$. Then there exists an open surjection $\mathscr{E} \stackrel{\gamma}{\rightarrow} \mathscr{S}$ such that each $S_{i}$ is countable in $\mathscr{E} ;$ i.e., for each $i$ there is an epimorphism $\mathbf{N} \rightarrow \gamma^{*}\left(S_{i}\right)$ in $\mathscr{E}$ ( $\mathscr{E}$ may be taken to be a space).

Proof. For a single object $S$, this is proved in Joyal and Tierney (1982, §V.3). The case of finite families $\left\{S_{1}, \ldots, S_{n}\right\}$ then follows, since open surjections are stable under composition and pullback. Finally, for the general case, one uses that open surjections are stable under filtered inverse limits (see Moerdijk (1984)).

Axioms of choice are generally not available in a topos. However, the following lemma says that we can apply an axiom of dependent choices in the context of toposes, provided we allow for a change of base. 
LeMMA C. Let $S$ be an object of $\mathscr{S}$, and let $T$ be an inhabited tree of finite sequences from $S$, "all whose branches are infinite":

(i) \langle\rangle$\in T$,

(ii) $u \leqslant v$ and $u \in T \Rightarrow v \in T$ ( $u \leqslant v$ means that $u$ extends $v$ ),

(iii) $u \in T \Rightarrow \exists s \in S u * s \in T$ (* for concatenation).

Then there exists an open surjection $\mathscr{E} \stackrel{\gamma}{\rightarrow} \mathscr{S}$ such that $T$ has a branch in $\mathscr{E}$; i.e., there is a function $\mathbf{N} \stackrel{\alpha}{\rightarrow} \gamma^{*}(S)$ in $\mathscr{E}$ such that (in $\mathscr{E}$ it holds that) $\forall n \in \mathbf{N}\langle\alpha(0), \ldots, \alpha(n)\rangle$ $\in \gamma^{*}(T)$.

Proof. We introduce a generic branch in the standard way: consider $T$ as a poset and make it into a presentation of a space $X$ by equipping it with the covering system generated by $\{u * s \mid s \in S\}$ covers $u$, for each $u \in T$. T has a top-element (i), and all covers are inhabited (iii), so if we take $\mathscr{E}$ to be the topos of sheaves on $X$, $\mathscr{E} \rightarrow \mathscr{S}$ is an open surjection.

\section{Proof of the theorem.}

2.1 Reduction to the case of spaces. As a first remark, let us point out that it suffices to prove the theorem stated in the introduction for the special case that $\mathscr{E}$ is the topos of sheaves on a space. Indeed, if $\mathscr{E}$ is a connected locally connected topos, there exists a connected locally connected map $X \rightarrow \mathscr{E}$, where $X$ is a connected locally connected space $(1.6$, Lemma $A)$. If the theorem is true for spaces, $X^{I} \rightarrow$ $X \times X$ is an open surjection. Since $X \times X \rightarrow \mathscr{E} \times \mathscr{E}$ is an open surjection, it then follows from Proposition V.1.2 of Joyal and Tierney (1984) that $\mathscr{E}^{I} \rightarrow \mathscr{E} \times \mathscr{E}$ must be one, provided we can show $X^{I} \rightarrow \mathscr{E}^{I}$ is a surjection.

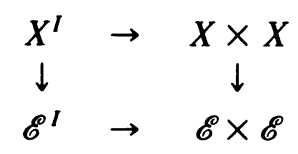

To this end, let us recall the construction of the map $X \rightarrow \mathscr{E}$ from Lemma A of 1.6 in more detail (Johnstone (1984)). In case $\mathscr{E}$ is the inhabited object classification $\mathscr{S}[U], X \rightarrow \mathscr{S}[U]$ is the classifier for a partial enumeration of this generic inhabited object $U$ of $\mathscr{S}[U]$. In other words, given a geometric morphism $\mathscr{F} \stackrel{f}{\rightarrow} \mathscr{S}[U]$, there is a 1-1 correspondence between maps $\mathscr{F} \rightarrow X$ over $\mathscr{S}[U]$ and partial enumerations of $f^{*}(U)$ in $\mathscr{F}$, i.e., diagrams in $\mathscr{F}$ of the form

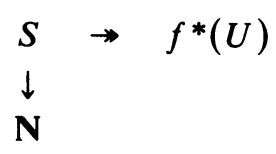

Write $X_{U}$ for this specific space $X$ (identified with the corresponding topos of sheaves), with a connected locally connected map $X_{U} \rightarrow \mathscr{S}[U]$.

For a general Grothendieck topos $\mathscr{E}$, there always is a spatial geometric morphism $\mathscr{E} \rightarrow \mathscr{S}[U]$, and the cover $X \rightarrow \mathscr{E}$ of 1.6 , Lemma $\mathrm{A}$, is simply constructed as the 
pullback

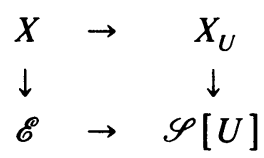

Claim. $X^{I} \rightarrow \mathscr{E}^{I}$ is a stable surjection.

Proof. It suffices to show that $\left(X_{U}\right)^{I} \rightarrow \mathscr{S}[U]^{I}$ is a stable surjection. We will indeed prove that for any base extension $\mathscr{G} \stackrel{p}{\rightarrow} \mathscr{S}$ and $\mathscr{G} \stackrel{\alpha}{\rightarrow} \mathscr{S}[U]^{I}$ there is an open surjection $\mathscr{H} \stackrel{q}{\rightarrow} \mathscr{G}$ and a lifting $\stackrel{\beta}{\beta} \rightarrow\left(X_{U}\right)^{I}$ with

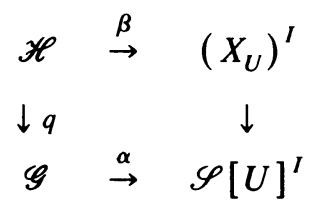

commutative.

By working "inside $\mathscr{G}$ ", we may assume $\mathscr{G}=\mathscr{S}(p=\mathrm{id})$. So $\stackrel{\alpha}{\rightarrow} \mathscr{S}[U]$, and this is just an inhabited sheaf $A$ on $I$ in $\mathscr{S}$, by definition of $\mathscr{S}[U]$. So the set of rational intervals $V$ with $\exists a \in A(V)$ cover $I$. A lifting $I \stackrel{\beta}{\rightarrow} X_{U}$ of $\alpha$ corresponds to a partial enumeration of $A$ in sheaves on $I$. To get this partial enumeration, we may extend the base (this is $q$ ): let $\mathscr{S}^{\prime} \rightarrow \mathscr{S}$ be an extension in which $\left\{V_{n}\right\}_{n}$ is the set of rational intervals in $I$ such that $A\left(V_{n}\right)$ is inhabited, and in which there are enumerations $\mathbf{N} \stackrel{e_{n}}{\rightarrow} A\left(V_{n}\right)$. (Such an open surjection $\mathscr{S}^{\prime} \rightarrow \mathscr{S}$ exists by Lemma $\mathrm{B}$ of 1.6.)

Now work in $\mathscr{S}^{\prime}$ : let $\Delta(\mathbf{N} \times \mathbf{N})$ denote the constant sheaf on $I$ (inside $\mathscr{S}^{\prime}$ ). Let $T \subset \Delta(\mathbf{N} \times \mathbf{N})$ be the subsheaf generated by $(n, m) \in T\left(V_{n}\right)$, all $n, m \in \mathbf{N}$. Then there is a map $T \stackrel{b}{\rightarrow} A$ of sheaves on $I$ whose component over $V_{n}$ is defined as

$$
b_{V_{n}}: T\left(V_{n}\right) \rightarrow A\left(V_{n}\right), \quad b_{V_{n}}(n, m)=e_{n}(m) .
$$

( $b$ is a well-defined map of sheaves, since the $V_{n}$ cover $I$, i.e., generate $I$ as a space.) $b$ is an enumeration of $A$ in $\operatorname{Sh}(I)$ and $T \subset \mathbf{N}$ in $\operatorname{Sh}(I)$ (up to coding $\mathbf{N} \stackrel{\sim}{\rightarrow} \mathbf{N} \times \mathbf{N}$ ), so $b$ corresponds to a geometric morphism $\beta$ such that

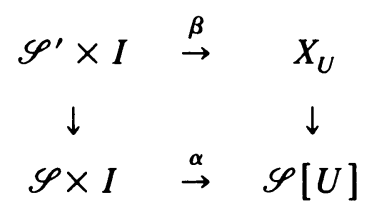

commutes. This proves the claim and completes 2.1 .

REMARK (ADDED IN PROOF). It was recently discovered that if $\mathscr{F} \rightarrow \mathscr{E}$ is any connected, locally connected map of toposes, then $\mathscr{F}^{I} \rightarrow \mathscr{E}^{I}$ is a stable surjection; cf. Moerdijk (1985). The proof of this result, however, is much more complicated than (and completely different from) the particular case treated in the preceding claim. 
We will first prove a slightly weaker version of the theorem, namely

2.2 Proposition. Let $X$ be a connected locally connected space. Then $X^{I} \rightarrow X \times X$ is a stable surjection.

As said in the introduction, our strategy will be to extend the base topos $\mathscr{S}$ sufficiently so as to be able to perform a classical argument (in 2.5 below) somewhat similar to Menger (1929), Moore (1932) (see also Engelking (1977, exercise 6.3.11)). To this end, we first introduce a generic pair of points (in 2.3), and then we force some countability conditions (in 2.4).

2.3 The generic pair of points. Let $X$ be a given connected locally connected space in $\mathscr{S}$. Let $\mathscr{F}=\operatorname{Sh}(X \times X) \stackrel{p}{\rightarrow} \mathscr{S}$ and write $Y=p^{\#}(X) . Y$ is a connected locally connected space in $\mathscr{F}$, and $p$ is an open surjection (in fact $p$ is connected locally connected). In $\mathscr{F}$, there is a generic pair of points

$$
\left(y_{0}, y_{1}\right): 1 \rightarrow Y \times Y=p^{\#}(X \times X),
$$

corresponding to the projections. A simple diagram argument shows that to prove that $X^{I} \rightarrow X \times X$ is a stable surjection in $\mathscr{S}$, it now suffices to find an open surjection $\mathscr{G} \stackrel{q}{\rightarrow} \mathscr{F}$ such that in $\mathscr{G}$ there is a map of spaces $I \stackrel{f}{\rightarrow} q^{\#}(Y)$ with $f(0)=x_{0}, f(1)=x_{1}(I$ is the formal unit interval in $\mathscr{G})$.

2.4 Countability conditions. Let $\mathbf{P}$ be the presentation by connected opens of $Y$ in $\mathscr{F}$, so $\mathbf{P}$ is a molecular presentation as in 1.3. For each $W \in \mathbf{P}$, let $\left\langle\mathscr{U}_{i}(W): i \in I_{W}\right\rangle$ be the family of covers of $W$ in $\mathbf{P}$. Adjoining surjective functions $\mathbf{N} \rightarrow I_{W}$ to $\mathscr{E}$ (for each $W \in \mathbf{P}$ ) as in 1.6, Lemma $\mathrm{B}$, we find an open surjection $\mathscr{F}^{\prime} \stackrel{r}{\rightarrow} \mathscr{F}$ such that in $\mathscr{F}^{\prime}, Z=r^{\#}(Y)$ has a molecular presentation in which for each element the family of "basic" covers of this element is countable.

Similarly, we can adjoin surjections $\mathbf{N} \rightarrow\left\{U \in \mathbf{P} \mid y_{0} \in U\right\}$ and $\mathbf{N} \rightarrow\left\{U \in \mathbf{P} \mid y_{1}\right.$ $\in U\}$. So in $\mathscr{F}^{\prime}$, the points $y_{0}, y_{1}$ of $r^{\#}(Y)$ each have a countable neighbourhood base consisting of elements of the molecular presentation.

So in $\mathscr{F}^{\prime}$, we now have the following data: a connected locally connected space $Z$ and two points $z_{0}, z_{1}$ of $Z$, with a molecular presentation $\mathbf{P}$ of $Z$ such that

(i) for all $W \in \mathbf{P},\left\langle\mathscr{U}_{n}(W): n \in \mathbf{N}\right\rangle$ enumerates the covers of $W$ in $\mathbf{P}$,

(ii) $\left\langle N_{n}\left(Z_{0}\right): n \in \mathbf{N}\right\rangle$ enumerates the elements of $\mathbf{P}$ which contain $z_{0}$,

(ii) $\left\langle N_{n}\left(z_{1}\right): n \in \mathbf{N}\right\rangle$ enumerates the elements of $\mathbf{P}$ which contain $z_{1}$.

2.5 Proof of Proposition 2.2. After these preparations, we can now construct an extension $\mathscr{G} \rightarrow \mathscr{F}^{\prime}$ such that in $\mathscr{G}$ there actually is a path $I \rightarrow s^{\#}(Z)$ from $z_{0}$ to $z_{1}$ ( $s$ will be an open surjection). We work in $\mathscr{F}^{\prime}$ with the data as in 2.4.

A chain from $z_{0}$ to $z_{1}$ is a chain $\left(V_{1}, \ldots, V_{k}\right)$ of elements of $\mathbf{P}$ (see 1.3) such that $z_{0} \in V_{1}$ and $z_{1} \in V_{k}$. Consider the tree $T$ of pairs of finite sequences $\left\langle\left(V_{1}^{m}, \ldots, V_{k(m)}^{m}\right)_{m \leqslant n},\left(\rho^{m}\right)_{m<n}\right\rangle$, where the $\left(V_{1}^{m}, \ldots, V_{k(m)}^{m}\right)$ are chains from $z_{0}$ to $z_{1}$, and the $\rho^{m}:\{1, \ldots, k(m+1)\} \rightarrow\{1, \ldots, k(m)\}$ are functions such that

(a) $j \leqslant j^{\prime} \Rightarrow \rho^{m}(j) \leqslant \rho^{m}\left(j^{\prime}\right)$, and $V_{j}^{m+1} \leqslant V_{\rho^{m}(j)}^{m}$;

(b) for each $m^{\prime}<m$ and each $j \leqslant k(m), V_{j}^{m}$ is contained in an element of $\mathscr{U}_{n}\left(V_{\rho^{m^{\prime}}}^{m^{\prime}} \ldots \ldots \rho^{m-1}(j)\right)$, for each $n \leqslant m$; 
(c) $V_{1}^{m}$ is contained in $N_{n}\left(z_{0}\right)$ for each $n \leqslant m$, and $V_{k(m)}^{m}$ is contained in $N_{n}\left(z_{1}\right)$ for each $n \leqslant m$;

(d) Given $i \leqslant k(m)$, suppose $\rho^{m}(j)=i$ for $j=j_{0}, j_{0}+1, \ldots, j_{0}+k$.

Then $V_{j_{0}}^{m+1} \leqslant V_{i-1}^{m}$ (unless $j_{0}=0$, i.e., $i=0$ ), and $V_{j_{0}+k}^{m+1} \leqslant V_{i+1}^{m}$ (unless $j_{0}+k$ $=k(m+1)$, i.e., $i=k(m))$.

It follows from the molecularity of $\mathbf{P}$ that any such pair of finite sequences satisfying (a)-(d) can be extended to a longer one. Explicitly: suppose we are given $\left\langle\left(V_{1}^{m}, \ldots, V_{k(m)}^{m}\right)_{m \leqslant n},\left(\rho^{m}\right)_{m<n}\right\rangle$ as above. Cover each $V_{j}^{n}(j=1, \ldots, k(n))$ by a common refinement $\mathscr{W}_{j}$ of the covers $\mathscr{U}_{n+1}\left(V_{\rho^{m \prime}}^{m^{\prime}} \ldots \ldots \circ \rho^{n-1}(j)\right), m^{\prime} \leqslant n$. Choose a $\tilde{W}_{0} \ni z_{0}$ in $\mathbf{P}$ such that $\tilde{W}_{0} \leqslant$ some element of $\mathscr{W}_{1}$, and $\tilde{W}_{0} \leqslant N_{n+1}\left(z_{0}\right)$. Similarly choose a $W_{k(n+1)} \ni z_{1}$ such that $W_{k(n+1)} \leqslant$ some element of $\mathscr{W}_{k(n)}$ and $W_{k(n+1)} \leqslant$ $N_{n+1}\left(z_{1}\right)$.

Now for each $j \leqslant k(n)$, some $W_{j} \in \mathscr{W}_{j}$ must have positive intersection with some $\tilde{W}_{j+1} \in \mathscr{W}_{j+1} \quad$ (i.e. $\exists U \in \mathbf{P} \quad U \leqslant W_{j}$ and $U \leqslant \tilde{W}_{j+1}$ ). Now let $V_{1}^{n+1}=\tilde{W}_{0}$, $V_{2}^{n+1}, \ldots, V_{k-1}^{n+1}=W_{1}$ be a chain in $\mathscr{W}_{1}$, and let $V_{k}^{n+1}$ be an element of $\mathbf{P}$ with $V_{k}^{n+1} \leqslant W_{1}$ and $V_{k}^{n+1} \leqslant \tilde{W}_{2}$ (so in particular $V_{k}^{n+1} \leqslant V_{1}^{n}$ and $V_{k}^{n+1} \leqslant V_{2}^{n}$, for condition (d)). Let $\rho^{n}(i)=1$ for $1 \leqslant i \leqslant k$. Now define the next bit $V_{k+1}^{n+1}, \ldots, V_{k+1}^{n+1}$ in a similar way: let $V_{k+1}^{n+1}=V_{k}^{n+1}$, let $V_{k+2}^{n+1}=\tilde{W}_{2}, V_{k+3}^{n+1}, \ldots, V_{k+1-1}^{n+1}=W_{2}$ be a chain from $\tilde{W}_{2}$ to $W_{2}$ in $\mathscr{W}_{2}$, and let $V_{k+1}^{n+1}$ be an element of $\mathbf{P}$ with $V_{k+1}^{n+1} \leqslant W_{2}$ and $V_{k+1}^{n+1} \leqslant \tilde{W}_{3}$. Let $\rho^{n}(i)=2$ for $k<i \leqslant k+l$; etc., until $V_{k(n+1)}^{n+1}:=W_{k(n+1)} \ni z_{1}$.

By Lemma $C$ of 1.6 , there is an open surjection $\mathscr{G} \rightarrow \mathscr{F}^{\prime}$ such that the tree $T$ has an infinite branch in $\mathscr{G}$. Replacing $\mathscr{F}^{\prime}$ by $\mathscr{G}$, we work within $\mathscr{G}$ with this fixed branch which we will denote by $\left\langle\left(V_{1}^{m}, \ldots, V_{k(m)}^{m}\right)_{m \in N},\left(\rho^{m}\right)_{m \in N}\right\rangle$.

We now mimic this branch of chains of $V_{j}^{m}$ 's by consecutive rational intervals in $I=[0,1]$. (Notational convention: the open interval $(p, q)$ stands for $[p, q)$ if $p=0$, and for $(p, q]$ if $q=1$.) Let $p_{0}^{-1}=0, q_{1}^{-1}=1$. Suppose we have defined $\left(p_{i}^{m}, q_{i}^{m}\right)$ for $1 \leqslant i \leqslant k(m)$,

$$
0=p_{1}^{m}<q_{1}^{m}=p_{2}^{m}<q_{2}^{m}=\cdots<q_{k(m)}^{m}=1 .
$$

Define $\left(p_{j}^{m+1}, q_{j}^{m+1}\right)$ for $1 \leqslant j \leqslant k(m+1)$ as follows. If $\rho^{m}(j)=i$ for $j=j_{0}, j_{0}+$ $1, \ldots, j_{0}+k$, choose rationals $r_{1}, \ldots, r_{k}$ with $p_{i}^{m}<r_{1}<\cdots<r_{k}<q_{i}^{m}$, and let

$$
p_{j_{0}}^{m+1}=p_{i}^{m}, \quad q_{j_{0}}^{m+1}=p_{j_{0}+1}^{m+1}=r_{1}, \ldots, q_{j_{0}+k-1}^{m+1}=p_{j_{0}+k}^{m+1}=r_{k}, \quad q_{j_{0}+k}^{m+1}=q_{i}^{m} .
$$

So for each $m,\left\{\left[p_{j}^{m}, q_{j}^{m}\right]: j=1, \ldots, k(m)\right\}$ is a "cover" of $[0,1]$ by consecutive closed intervals having one point in common, and the cover for $m+1$ refines the one for $m$ according to the function $\rho^{m}$.

Define a function $f^{*}: \mathbf{P} \rightarrow \mathcal{O}(I)$ by

$$
f^{*}(U)=\bigvee\left\{\left(p_{j}^{m}, q_{j^{\prime}}^{m}\right) \mid j \leqslant j^{\prime} \text { and } V_{j}^{m} \vee \cdots \vee V_{j^{\prime}}^{m} \leqslant U\right\}
$$

We claim that $f^{*}$ defines a continuous map of spaces $I \stackrel{f}{\rightarrow} Z$, and that $f(0)=z_{0}$, $f(1)=z_{1}$.

Proof of this claim:

(i) $f^{*}(1)=\left(p_{0}^{0}, q_{k(0)}^{0}\right)=[0,1]$ (by the notational convention). 
(ii) $f^{*}$ preserves binary meets; more precisely, since $\mathbf{P}$ does not have meets, if $W \leqslant f^{*}(U)$ and $\tilde{W} \leqslant f^{*}(\tilde{U})$ then $W \wedge \tilde{W} \leqslant f^{*}(V)$ for some $V \in \mathbf{P}$ with $V \leqslant U$, $V \leqslant \tilde{U}$. Indeed, suppose $W=\left(p_{j}^{m}, q_{j^{\prime}}^{m}\right) \leqslant f^{*}(U)$ because $V_{j}^{m} \vee \cdots \vee V_{j^{\prime}}^{m} \leqslant U$, and $\tilde{W}=\left(p_{j}^{\tilde{m}}, q_{j}^{\tilde{m}}\right) \leqslant f^{*}(\tilde{U})$ because $V_{\tilde{j}}^{\tilde{m}} \vee \cdots \vee V_{\tilde{j}^{\prime}}^{\tilde{m}} \leqslant \tilde{U}$. Let us say $\tilde{m} \geqslant m$, and $p_{j}^{m}<p_{j}^{\tilde{m}_{i}}<q_{j^{\prime}}^{m} \leqslant p_{j^{\prime}}^{\tilde{m}}$ (other cases are symmetric or trivial). By construction, $q_{j^{\prime}}^{m}=q_{i}^{\tilde{m}}$ for some $i \geqslant \tilde{j}$, and

$$
V_{\tilde{j}}^{\tilde{m}} \vee \cdots \vee V_{i}^{\tilde{m}} \leqslant V_{j}^{m} \vee \cdots \vee V_{j^{\prime}}^{m} \leqslant U .
$$

So $W \wedge W^{\prime}=\left(p_{j}^{\tilde{m}}, q_{i}^{\tilde{m}}\right) \leqslant f^{*}\left(V_{j}^{\tilde{m}} \vee \cdots \vee V_{i}^{\tilde{m}}\right)$, and $V_{j}^{\tilde{m}} \vee \cdots \vee V_{i}^{\tilde{m}} \in \mathbf{P}$ since $\mathbf{P}$ is closed under sups of chains, and moreover $V_{j}^{\tilde{m}} \vee \cdots \vee V_{i}^{\tilde{m}} \leqslant$ both $U$ and $\tilde{U}$.

(iii) $f^{*}$ maps basic covers in $\mathbf{P}$ to sups in $\mathcal{O}(I)$ : Let $\left\{U_{\alpha}\right\}_{\alpha}$ be a cover of $U \in \mathbf{P}$, and suppose $\left(p_{j}^{m}, q_{j^{\prime}}^{m}\right) \leqslant f^{*}(U)$ because $V_{j}^{m} \vee \cdots \vee V_{j^{\prime}}^{m} \leqslant U$. For each $k, j \leqslant k \leqslant$ $j^{\prime}$, we have by stability a cover $\left\{W_{\beta}^{k}\right\}$ of $V_{k}^{m}$ in $\mathbf{P}$ such that each $W_{\beta}^{k} \leqslant$ some $U_{\alpha}$. Say $\left\{W_{\beta}^{k}\right\}=\mathscr{U}_{n_{k}}\left(V_{k}^{m}\right)$. Let $\tilde{m}=\max \left(n_{j}, \ldots, n_{j^{\prime}}\right)$. Now consider the chain $\left(V_{1}^{\tilde{m}}, \ldots, V_{k(\tilde{m})}^{\tilde{m}}\right)$ from $z_{0}$ to $z_{1}$. By definition, there are $1 \leqslant l_{k} \leqslant l_{k}^{\prime} \leqslant k(\tilde{m})$ such that

$$
\rho^{m} \circ \cdots \circ \rho^{\tilde{m}-1}(i)=k \Leftrightarrow l_{k} \leqslant i \leqslant l_{k}^{\prime}
$$

(for $k=1, \ldots, k(m)$, but only $k=j, \ldots, j^{\prime}$ are relevant). So for $l_{k} \leqslant i \leqslant l_{k}^{\prime}$,

$$
V_{i}^{\tilde{m}} \leqslant \text { some } W_{\beta}^{k} \leqslant \text { some } U_{\alpha} \text {. }
$$

Hence $\left(p_{i}^{\tilde{m}}, q_{i}^{\tilde{m}}\right) \leqslant f^{*}\left(U_{\alpha}\right)$, and therefore

$$
\left(p_{l_{j}}^{\tilde{m}}, q_{l_{j}}^{\tilde{m}}\right) \vee \cdots \vee\left(p_{l_{j^{\prime}}}^{\tilde{m}}, q_{l_{j^{\prime}}}^{\tilde{m}}\right) \leqslant \bigvee_{\alpha} f^{*}\left(U_{\alpha}\right)
$$

Since $p_{j}^{n}=p_{l_{i}}^{\tilde{m}}$ and $q_{j^{\prime}}^{m}=q_{l^{\prime}}^{\tilde{m}}$, this almost means that $\left(p_{j}^{m}, q_{j^{\prime}}^{m}\right) \leqslant \bigvee_{\alpha} f^{*}\left(U_{\alpha}\right)$, but we miss the boundary points! To make up for those, however, it suffices to note the following consequence of condition (d): Given any $V_{i}^{n}$, there is an $n^{\prime} \geqslant n$ such that $V_{j^{\prime}}^{n}, \ldots, V_{j^{\prime}}^{n^{\prime}} \leqslant V_{i}^{m}$ and $\rho(j)<i<\rho\left(j^{\prime}\right)$, where $\rho=\rho^{n} \circ \ldots \circ \rho^{n^{\prime}-1}$ (i.e., for chains which are sufficiently fine, we get over the boundary).

This completes the proof that $f^{*}$ defines a map $f: I \rightarrow Z$ of spaces.

(iv) Finally, $f(0)=z_{0}, f(1)=z_{1}$ : Clearly, if $f(0) \in U$ then $V_{0}^{m} \leqslant U$ for some $m$, so $z_{0} \in U$. Conversely, if $z_{0} \in U$ then $U=N_{n}\left(z_{0}\right)$ for some $n$, so $V_{0}^{m} \leqslant U$ for $m \geqslant n$; hence $f(0) \in U$. Thus $f(0)=z_{0}$ as points of $Z$. Similarly $f(1)=z_{1}$.

This completes the proof of Proposition 2.2. In 2.7 we will show that $X^{I} \rightarrow X \times X$ is in fact an open surjection.

2.6 REMARK. As said in the introduction, one can also give a more "algebraic" proof, by working directly with sites (presentations). We briefly describe the sites involved. Let $X$ be a connected locally connected space, with a molecular presentation P. Hyland (1981) gives a presentation for the space $X^{I}$. It is not hard to see that in the present case, it suffices to consider elements in the presentation of the form $\bigwedge_{i=1}^{n}\left[\left(p_{i}, p_{i+1}\right), U_{i}\right]$, where $0=p_{1}<\cdots<p_{n}=1$ are rationals, and $\left(U_{1}, \ldots, U_{n}\right)$ is a chain in $\mathbf{P}$ (Hyland would write $\left[\left(p_{i}, p_{i+1}\right)<<f^{*}\left(U_{i}\right)\right]$ for our $\left.\left[\left(p_{i}, p_{i+1}\right), U_{i}\right]\right)$. Let $\mathbf{Q}$ be a presentation of $X^{I}$ with underlying poset consisting of opens of $X^{I}$ of this form. Let $\mathbf{P} \otimes \mathbf{P}$ denote the presentation of $X \times X$ obtained in the obvious way from the presentation $\mathbf{P}$ of $X$. The inverse image $\mathcal{O}(X) \otimes \mathcal{O}(X) \stackrel{F}{\rightarrow} \mathcal{O}\left(X^{I}\right)$ of the 
map $X^{I} \rightarrow X \times X$ of Proposition 2.2 is induced by the functor

$$
F(V \otimes W)=\bigvee\left\{\bigwedge_{i=1}^{n}\left[\left(p_{i}, p_{i+1}\right), U_{i}\right] \in \mathbf{Q} \mid U_{1} \leqslant V, U_{n} \leqslant W\right\} .
$$

To show that $F$ induces an open surjection, one would have to prove that $\mathcal{O}(X \times X) \stackrel{F}{\rightarrow} \mathcal{O}\left(X^{I}\right)$ has a left inverse, left adjoint $G: \mathcal{O}\left(X^{I}\right) \rightarrow \mathcal{O}(X \times X)$ described in terms of presentations by

$$
\begin{gathered}
\mathbf{Q} \stackrel{G}{\rightarrow} \mathbf{P} \otimes \mathbf{P}, \\
G\left(\bigwedge_{i=1}^{n}\left[\left(p_{i}, p_{i+1}\right), U_{i}\right]\right)=U_{1} \otimes U_{n},
\end{gathered}
$$

and that the Frobenius law $G(U \wedge F(V))=G(U) \wedge V$ holds. The proof, however, would be less intuitive, and at least as complicated, as our "geometric" proof.

2.7 Openness of the map $X^{I} \rightarrow X \times X$. Given the fact that $X^{I}$ has a presentation $\mathbf{Q}$ as in 2.6, our proof of 2.2 actually shows that $X^{I} \rightarrow X \times X$ is an open surjection. We argue again in the geometric style, using base extensions to enable ourselves to reason about points.

In general, a map $B \stackrel{f}{\rightarrow} A$ of spaces in $\mathscr{S}$ (or in any topos) is open iff the image $f(V)$ is an open subspace of $A$ for all $V$ in some basis (some presentation) of $B$ (see Joyal and Tierney (1984)). If we allow for change of base, images can be described in terms of points, just as in topology: if $V \in \mathcal{O}(B)$ and $U \in \mathcal{O}(A)$, then $f(V)=U$ iff for any geometric morphism $\mathscr{G} \rightarrow \mathscr{S}$ and any point $p \in \varphi^{\#}(A)$, we have (writing $V$ for $\varphi^{\#}(V), U$ for $\left.\varphi^{\#}(U)\right)$

$$
\begin{aligned}
p \in U \Leftrightarrow & \text { there is a surjection } \mathscr{H} \stackrel{\psi}{\rightarrow} \mathscr{G} \text { and a point } \\
& q \in V \subset \psi^{\#} \varphi^{\#}(B) \text { such that in } \mathscr{H}, p=f(q) .
\end{aligned}
$$

Let us consider the special case where $B \stackrel{f}{\rightarrow} A$ is the map $X^{I} \rightarrow X \times X$ of Proposition 2.2. Take a basic open $U=\Lambda_{i=1}^{r}\left[\left(p_{i}, p_{i+1}\right), U_{i}\right]$ of $X^{I}$ as in 2.6. We claim that the image of $U$ is the open subspace $U_{1} \times U_{n}$ of $X \times X$. To show the equivalence (*), choose $\mathscr{G} \stackrel{\varphi}{\rightarrow} \mathscr{S}$ and a point $p=\left(x_{0}, x_{1}\right) \in \varphi^{\#}\left(U_{1}\right) \times \varphi^{\#}\left(U_{n}\right)$ in $\mathscr{G}$. Since $\left(\varphi^{\#}\left(U_{1}\right), \ldots, \varphi^{\#}\left(U_{n}\right)\right)$ is a chain in $\mathscr{G}$, there is an open surjection $\mathscr{G}_{0} \rightarrow \mathscr{G}$ such that, writing $\varphi_{0}$ for the composite $\mathscr{G}_{0} \rightarrow \mathscr{G} \stackrel{\varphi}{\rightarrow} \mathscr{S}$, there are points $y_{i} \in \varphi_{0}^{\#}\left(U_{i} \wedge U_{i+1}\right)=$ $\varphi_{0}^{\#}\left(U_{i}\right) \wedge \varphi_{0}^{\#}\left(U_{i+1}\right) \quad(i=1, \ldots, n-1)$. Let $y_{0}=x_{0}, y_{n}=x_{1}$ in $\mathscr{G}_{0}$. Since each $\varphi_{0}^{\#}\left(U_{i}\right)$ is a connected locally connected space in $\mathscr{G}_{0}$, our proof of 2.2 shows that there exists an open surjection $\mathscr{H}^{\psi} \stackrel{\psi}{\rightarrow} \mathscr{G}_{0}$ such that in $\mathscr{H}$ there are paths $f_{i}$ : $I \rightarrow \psi^{\#} \varphi_{0}^{\#}\left(U_{i}\right)$ with $f_{i}(0)=y_{i}, f_{i}(1)=y_{i+1}(i=0, \ldots, n-1)$. Putting these paths together, we obtain a map $I \rightarrow \psi^{\#} \varphi_{0}^{\#}(X)$ with $f(i / n)=y_{i}(i=0, \ldots, n)$. This shows $\Rightarrow$ of $(*)$ for this particular case. 
The other implication $\Leftarrow$ is obvious.

This completes the proof of the theorem as stated in the introduction.

2.8 REMARK. Finally, we point out that openness of the map $\mathscr{E}^{I} \rightarrow \mathscr{E} \times \mathscr{E}$ can be of interest, even if this map is not surjective. In fact, this generalizes the notion of semilocal path-connectedness for topological spaces: one easily shows that for a topological space $X$, the map $X^{I} \rightarrow X \times X$ (of topological spaces, not of toposes) is open iff $X$ is semilocally path-connected. (We are indebted to P. T. Johnstone for this observation.)

\section{REFERENCES}

M. Barr and R. Paré, Molecular toposes, J. Pure Appl. Algebra 17 (1980).

R. Engelking, General topologv, PWN, Warsaw, 1977.

M. Fourman and R. Grayson, Formal spaces, The Brouwer Centenary Symposium (Eds. A. S. Troelstra and D. van Dalen), North-Holland, Amsterdam, 1982.

A. Grothendieck and J. L. Verdier, Théorie des topos et cohomologie étale des schémas (SGA 4), Lecture Notes in Math., vols. 269 and 270, Springer, 1972.

M. Hyland, Function spaces in the category of locales, Continuous Lattices, Lecture Notes in Math., vol. 871, Springer, 1981.

J. R. Isbell, Atomless parts of spaces, Math. Scand. 31 (1972).

P. T. Johnstone, Open maps of toposes, Manuscripta Math. 31 (1980). , Stone spaces, Cambridge Univ. Press, 1982.

. How general is a generalized space? Aspects of Topology, in memory of H. Dowker, LMS Lecture Notes 93, Cambridge Univ. Press, 1985.

P. T. Johnstone and A. Joyal, Continuous categories and exponential toposes, J. Pure Appl. Algebra 25 (1982).

A. Joyal and M. Tierney, An extension of the Galois theory of Grothendieck, Mem. Amer. Math. Soc. 309 (1984).

K. Menger, Über die Dimension von Punktmengen III: zur Begründung einer axiomatischen Theorie der Dimension, Monatsh. Math. Phys. 36 (1929).

I. Moerdijk, Continuous fibrations and inverse limits of toposes (preprint 1984), Compositio Math. (to appear).

, Path-lifting for Grothendieck toposes (preprint 1985) (submitted).

R. L. Moore, Foundations of point set theory, Amer. Math. Soc. Colloq. Publ., vol. 13, Amer. Math. Soc., Providence, R. I., 1932.

L. A. Steen and J. A. Seebach, Counterexamples in topology, 2nd ed., Springer, 1978.

Mathematisch InStituUt, Universiteit VAN AMSTERDam, AmSTERDAM, The NeTHERLANDS

Mathematics Division, University of SusSex, SusSex, ENGLAND 